\title{
A Patient with Septo-Optic Dysplasia Accompanying with Central Precocious Puberty
}

\section{Shuntaro Morikawa and Toshihiro Tajima*}

Department of Pediatrics, Hokkaido University School of Medicine, N15, W7, Sapporo, Japan

\begin{abstract}
Septo-Optic Dysplasia (SOD) is a heterogeneous condition defined by any combination of i) optic nerve hypoplasia, ii) hypopituitarism, and iii) midline brain anomalies such as agenesis of the corpus callosum and absence of the septum pellucidum. We encountered a Japanese girl with optic nerve hypoplasia, growth failure, precocious puberty, and mild developmental delay. Her optic nerve hypoplasia was diagnosed at 4 months of age. Thereafter, she showed growth failure from infancy. Gradual breast development was noticed from 6 years of age. Therefore, she was referred to us at 9.2 years of age. Endocrinological investigations demonstrated deficiencies in growth hormone, adrenocorticotropic hormone, and cortisol. However, serum levels of luteinizing hormone, follicle stimulating hormone, and estradiol were increased to pubertal levels. Brain MRI revealed bilateral optic nerve hypoplasia, a normal anterior pituitary lobe, an invisible pituitary stalk, and an invisible posterior lobe. Based on these findings, she was diagnosed as having SOD with growth hormone deficiency, secondary adrenal insufficiency, and central precocious puberty. Replacement of hydrocortisone, recombinant growth hormone replacement, and suppression therapy by gonadotropin releasing hormone analog were initiated. Her growth rate and physical activity were improved after treatment. While SOD is rare, children with optic nerve hypoplasia should be carefully checked for symptoms of pituitary hormone deficiency. Timely diagnosis of endocrine deficits could reduce the morbidity of patients with SOD.
\end{abstract}

Keywords: Septo-optic dysplasia (SOD); Pituitary; Growth hormone deficiency; Precocious puberty

\section{Case Presentation}

The patient is a Japanese girl, currently 10.6 years old. She was born after 40 weeks of gestation by normal vaginal delivery and was the first child of non-consanguineous parents. The patient had no siblings, and her parents were healthy. Her birth weight was $2900 \mathrm{~g}$ (-0.3 SD from the mean for normal Japanese girls) and length $48.7 \mathrm{~cm}(-0.4$ SD from the mean for normal Japanese girls). At 1 month of age, she did not show tracking eye movement. Fundus examination led to the diagnosis of bilateral optic nerve hypoplasia at 4 months of age. As shown by her growth chart (Figure 1), she showed short stature (below -2.5 SD from the mean for normal Japanese girls) from infancy, but investigations for short stature were not done. Her psychomotor development was mildly delayed. Her parents noticed that her breasts developed from 6.5 years of age, and she manifested menarche at 9 years of age. Therefore, she was referred to our hospital at 9 years and 2 months of age. On physical examination, her height was $119.3 \mathrm{~cm}(-2.2 \mathrm{SD}$ from the mean for normal Japanese girls) and her weight was $19.0 \mathrm{~kg}(-1.2 \mathrm{SD}$ from the mean for normal Japanese girls). Her breast development was at Tanner stage IV and her pubic hair was at Tanner stage I. She did not show polydipsia or polyuria.

Her bone age at 9 years and 2 months was advanced (Japanese TW2 method, bone age of 10 years and 8 months). Her morning urine specific gravity was 1.010 . Her basal luteinizing hormone (LH) and Follicle Stimulating Hormone (FSH) levels were $2.4 \mathrm{mIU} / \mathrm{ml}$ and $4.6 \mathrm{mIU} /$ $\mathrm{ml}$, respectively, representing pubertal levels. Her serum estradiol was also at a pubertal level $(18.0 \mathrm{pg} / \mathrm{ml})$. Stimulation tests showed growth hormone $(\mathrm{GH})$, adrenocorticotropic hormone (ACTH), and cortisol deficiencies (Table 1). TSH response to TRH seemed blunted. Serum free $\mathrm{T} 4$ level was $1.35 \mathrm{ng} / \mathrm{dl}$. Brain magnetic resonance imaging (MRI) revealed a normally sized anterior pituitary (Figure 2A); however, a pituitary stalk and posterior lobe were not detected (Figure 2A). In addition, dysgenesis of the bilateral optic nerve was found (arrow in Figure 2B). Pelvic MRI identified a mature uterus and $1.5 \mathrm{~cm} \times 1.5 \mathrm{~cm}$ sized bilateral ovaries. Based on these findings, she was diagnosed with

\begin{tabular}{|l|l|l|l|l|l|l|}
\hline & 0 min & 15 min & 30 min & 60 min & 90 min & 120 min \\
\hline GH $(\mathrm{ng} / \mathrm{ml})$ & 1.43 & 1.55 & 2.33 & 2.16 & 2.55 & 2.11 \\
\hline TSH $\square$ U $/ \mathrm{ml})$ & 1.35 & 7.18 & 8.53 & 6.00 & 4.50 & 3.63 \\
\hline PRL $(\mathrm{ng} / \mathrm{ml})$ & 14.5 & 77.9 & 65.4 & 40.1 & 24.3 & 22.0 \\
\hline ACTH $(\mathrm{pg} / \mathrm{ml})$ & 35.01 & 32.39 & 27.69 & 28.63 & 26.70 & 23.23 \\
\hline CS $\square \mathrm{g} / \mathrm{dl})$ & 8.8 & 8.4 & 7.7 & 7.1 & 5.5 & 5.4 \\
\hline
\end{tabular}

$\mathrm{GH}$ provocative tests were performed using insulin $(0.1 \mathrm{IU} / \mathrm{kg})$. Serum levels of thyroid stimulating hormone (TSH) and prolactin (PRL) were determined in response to thyrotropin-releasing hormone $(10 \mu \mathrm{g} / \mathrm{kg})$. Plasma adrenocorticotropic hormone (ACTH) and serum cortisol were determined after an insulin tolerance test.

Table 1: Results of insulin and thyrotropin-releasing hormone stimulation tests.

SOD accompanying GH deficiency, secondary adrenal insufficiency, and central precocious puberty.

Replacement therapy with growth hormone $(0.175 \mathrm{mg} / \mathrm{kg} /$ week $)$, hydrocortisone (10 $\mathrm{mg} /$ day), and suppression therapy for central precocious puberty using gonadotropin releasing hormone $(\mathrm{GnRH})$ analog $(0.09 \mathrm{mg} / \mathrm{kg} / \mathrm{month})$ were started. Her growth rate was improved (Figure 1). Based on interviews with her mother, the physical activity of the patient was also improved. At her present age of 10.6 years, her body weight is $22 \mathrm{~kg}$ (-1.2 SD from the mean for normal Japanese girls) and height $127.2 \mathrm{~cm}$ (-1.7 SD from the mean for normal Japanese girls). Her breast development is currently at Tanner stage III, and LH and FSH have been suppressed to prepubertal levels. Her free T4 level after

*Corresponding author: Toshihiro Tajima, Department of Pediatrics, Hokkaido University School of Medicine, N15, W7, Sapporo, Japan, Tel: +81 11-716-2111; E-mail: tajeari@hokudai.med.ac.jp

Received August 29, 2015; Accepted September 10, 2015; Published September 17, 2015

Citation: Morikawa S, Tajima T (2015) A Patient with Septo-Optic Dysplasia Accompanying with Central Precocious. J Clin Case Rep 5: 587. doi:10.4172/21657920.1000587

Copyright: (c) 2015 Morikawa S, et al. This is an open-access article distributed under the terms of the Creative Commons Attribution License, which permits unrestricted use, distribution, and reproduction in any medium, provided the original author and source are credited. 
Citation: Morikawa S, Tajima T (2015) A Patient with Septo-Optic Dysplasia Accompanying with Central Precocious. J Clin Case Rep 5: 587. doi:10.4172/2165-7920.1000587

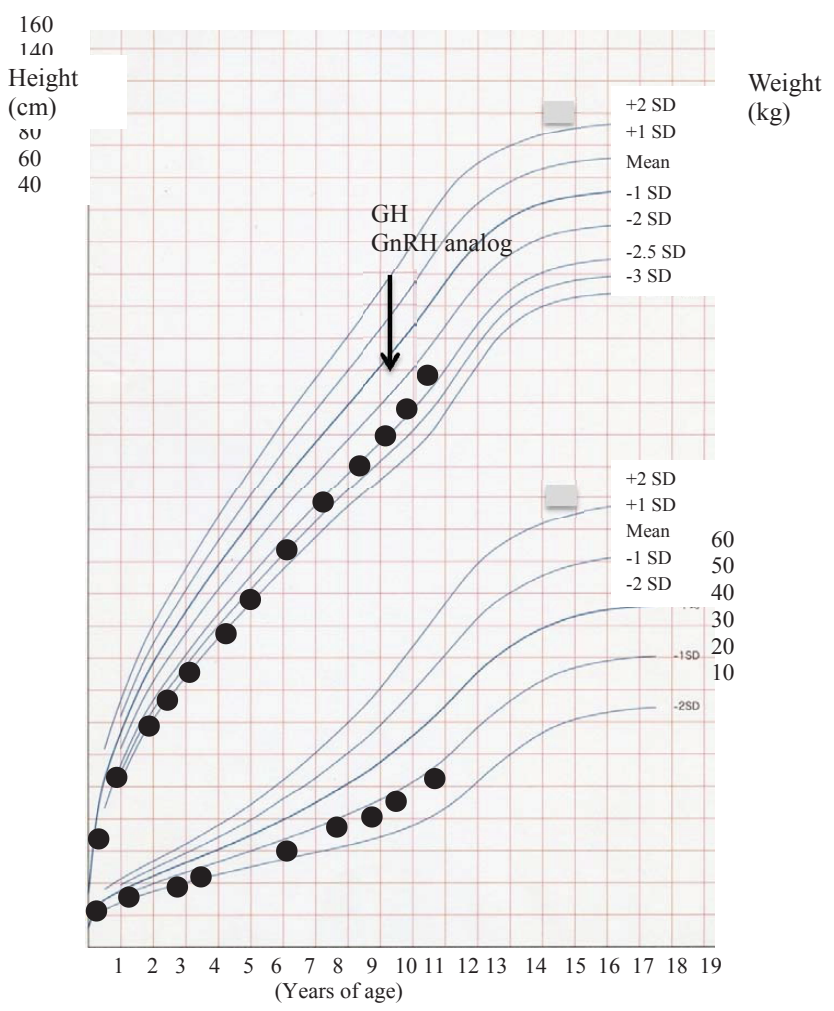

Figure 1: Growth chart of the patient.

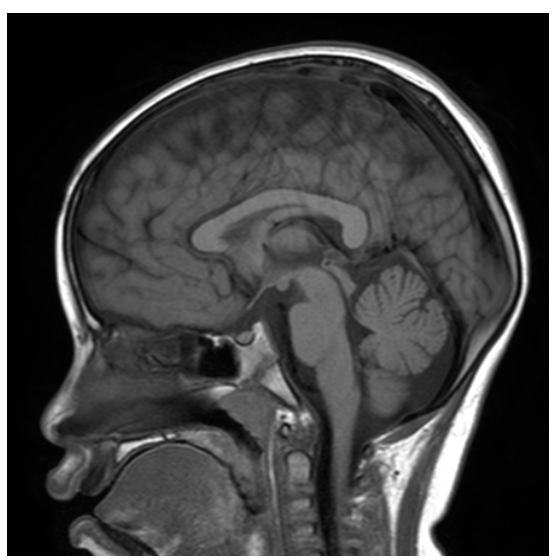

Figure 2A: MRI displayed a normal anterior pituitary lobe (arrow), but an invisible pituitary stalk and posterior pituitary lobe (arrowhead).

GH replacement therapy was within normal range. She has not shown polyuria or polydipsia so far.

\section{Discussion}

Septo-Optic Dysplasia (SOD), also known as de Morsier syndrome, is a rare congenital disease. Diagnosis of SOD is made based on the presence of 2 or more features of the classic triad: (i) optic nerve hypoplasia, (ii) hypopituitarism, ranging from isolated to multiple hormone deficiency, and (iii) midline brain defects (1). However, SOD is heterogeneous and may accompany other brain anomalies and developmental delay [1,2]. Regarding pubertal disturbance, patients with SOD usually show gonadotropin deficiency as a manifestation

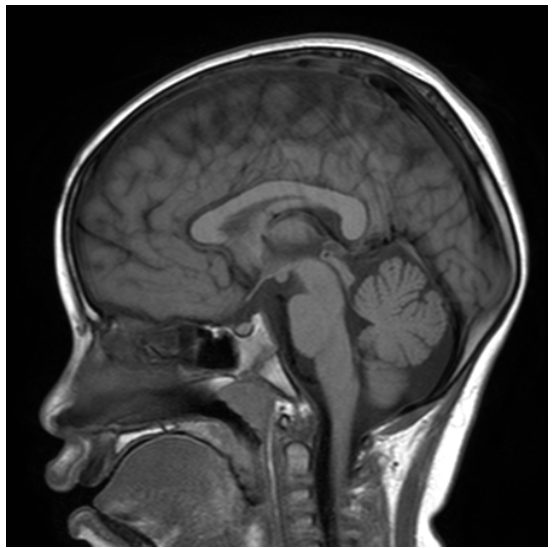

Figure 2A: MRI displayed a normal anterior pituitary lobe (arrow), but an invisible pituitary stalk and posterior pituitary lobe (arrowhead).

of hypopituitarism; however, it has been reported that several patients showed central precocious puberty [3]. Here we report a Japanese girl with SOD accompanying Growth Hormone (GH) deficiency, Adrenocorticotropic Hormone (ACTH) deficiency, and central precocious puberty. The incidence of SOD has been reported with 1 in 10,000 births, equally prevalent in males and females. The diagnosis of SOD is based on the presence of two or more features of the classical triad of optic nerve hypoplasia, pituitary hormone abnormalities, and midbrain defects such as agenesis of the corpus callosum and absence of the septum pellucidum [1]. As our patient showed optic nerve hypoplasia and pituitary dysfunction, a diagnosis of SOD was settled.

Cerebellar hypoplasia, schizencephaly, aplasia of the fornix, encephalocele, and cortical dysplasia were also reported in SOD patients [1-3]. In addition, neurological symptoms are frequently observed, including mental retardation, epilepsy, and communicative or behavioral difficulties [1]. In this patient, no anomalies of the cerebrum were identified; however, she showed mild mental retardation. This is presumably related to SOD. The most common hormone deficiency is GH alone or in combination with deficiencies in other pituitary hormones [1-4]. Secondary adrenal insufficiency due to ACTH deficiency has also been reported in SOD patients. From her past history, she did not have any known episodes of adrenal crisis. However, it has been reported that the degree of ACTH deficiency can gradually progress in congenital combined pituitary hormone deficiency [5]. Moreover, Atapattu et al. [4] reported that among 227 patients with SOD, 3 patients died from adrenal crisis. Therefore, the evaluation of the hypothalamic-pituitary-adrenal system is mandatory in patients with SOD. When secondary adrenal insufficiency is diagnosed, hydrocortisone replacement should be done immediately. The patient also showed central precocious puberty in addition to $\mathrm{GH}$ and ATCH deficiency. In SOD, both gonadotropin deficiency and central precocious puberty have been reported [3,6-8]. Central precocious puberty usually includes acceleration of the growth rate and GH deficiency usually shows growth failure. In this patient growth acceleration due to precocious puberty could mask growth hormone deficiency. The simultaneous occurrence of central precocious puberty and GH deficiency is a further risk for short stature [9]. It is thought there is little effect of treating girls aged over 8 years and with only central precocious puberty using GnRH analog for the purposes of increasing adult height [10]. However, as our patient had GH deficiency, the use of $\mathrm{GnRH}$ analog together with $\mathrm{GH}$ replacement was rationalized. 
Citation: Morikawa S, Tajima T (2015) A Patient with Septo-Optic Dysplasia Accompanying with Central Precocious. J Clin Case Rep 5: 587. doi:10.4172/2165-7920.1000587

This case emphasizes the importance of evaluating growth in children with optic nerve hypoplasia. When pediatricians suspect an endocrine deficit, laboratory investigations including pituitary function tests and brain MRI should be performed. Timely diagnosis of endocrine deficits and adequate replacement of deficient hormones could decrease the morbidity and improve the health of patients with these conditions.

\section{References}

1. McCabe MJ, Alatzoglou KS, Dattani MT (2011) Septo-optic dysplasia and othe midline defects: the role of transcription factors: HESX1 and beyond. Best Pract Res Clin Endocrinol Metab 115-124.

2. Birkebaek NH, Patel L, Wright NB, Grigg JR, Sinha et al. (2003) Endocrine status in patients with optic nerve hypoplasia: relationship to midline central nervous system abnormalities and appearance of the hypothalamic-pituitary axis on magnetic resonance imaging. J Clin Endocrinol Metab 88: 5281-5286.

3. Oatman OJ, McClellan DR, Olson ML, Garcia-Filion P (2015) Endocrine and pubertal disturbances in optic nerve hypoplasia, from infancy to adolescence. Inter J Pediatr Endocrinol.
4. Atapattu N, Ainsworth J, Willshaw H, Parulekar M, MacPherson L et al. (2012) Septo-optic dysplasia: antenatal risk factors and clinical features in a regional study. Horm Res Paediatr 78:81-87

5. Takagi M, Ishii T, Inokuchi M, Amano N, Narumi S et al. (2012) Gradual loss of ACTH due to a novel mutation in LHX4: comprehensive mutation screening in Japanese patients with congenital hypopituitarism. PLoS One 7: e46008.

6. Huseman C, Kelch R, Hopwood N, Zipf W (1978) Sexual precocity associated with optic dysplasia and hypothalamic hypopituitarism. J Pediatr 92: 748.

7. Margalith D, Tze WJ, Jan JE (1985) Congenital optic nerve hypoplasia with hypothalamic-pituitary dysplasia. A review of 16 cases. Am J Dis Child 139 : 361-366.

8. Mohney BG, Young RC, Diehl N (2013) Incidence and associated endocrine and neurologic abnormalities of optic nerve hypoplasia. JAMA Ophthalmology 131: 898-902.

9. Ladjouze A, Soskin S, Garel C, Jullien M, Naud-Saudreau C et al. (2007) GH deficiency with central precocious puberty: a new rare disorder associated with a developmental defect of the hypothalamic-pituitary area. Eur J Endocrinold 156: 463-469.

10. Fuqua JS (2013) Treatment and Outcomes of Precocious Puberty: An Update. $\mathrm{J}$ Clin Endocrinol Metab 98: 2198-2207 